\title{
Application of Magnetohydrodynamic Effect to the Analysis of Electrochemical Reactions-1. MHD Flow of an Electrolyte Solution in an Electrode-Cell with a short Rectangular Channel
}

\author{
Ryoichi AOGAKI*, Kazuo FUEKI* and Takashi MUKAIBO*
}

\begin{abstract}
Imposition of magnetic field on an electrode system causes a flow of electrolyte solution. The effect of this induced flow was quantitatively analysed from the aspects of magnetohydrodynamics for a cell equipped with a pair of parallel electrodes immersed in an electrolyte solution. Observed flow rates were in good agreement with calculated ones.
\end{abstract}

\section{Introduction}

It has been reported that when an external magnetic field is imposed on an electrolytic current system, the electromagnetic force induces a flow of the solution and enhances the rate of the diffusional mass transfer ${ }^{12}$ (4). However, quantitative analysis has not been attempted to determine the flow rate of the solution. In order to utilize this magnetohydrodynamic $(m h d)$ flow of the electrolyte solution as a mean for the analysis of electrochemical reactions, it is required to obtain the information on the solution flow in potential flow mode which is encountered with such electrode system as in this study, as will be mentioned later.

In this paper, we will first represent the flow rate of the electrolyte solution in terms of the externally applied magnetic field, electrolytic current and cell configuration. Then the observed flow rate is compared with the calculated one. Finally, the possible application of the system is discussed as a mean for controlling the bulk motion of electrolyte solution which is essential to a steady state control of the thickness of the diffusion layer on electrodes during electrochemical measurements.

* Department of Industrial Chemistry, Faculty of Engineering, University of Tokyo (Hongo, Bunkyoku, Tokyo)

\section{Theoretical}

\subsection{Main flow induced by electromagne- tic force}

Figure 1 shows the schematic configuration of electrode cell used for this study. The cell is composed of a short rectangular channel with two open ends. Inside the channel, a pair of electrodes a are embedded on the top and bottom walls, face to face. A reference electrode $b$, when used, is inserted into a drilled small hole from the back of the working electrode by the use of conical Luggin capillary so that the tip of the capillary comes to the same level of the inner surface of the electrode. At the entrance of the channel, another pair of electrodes $\mathrm{e}$ are equipped to measure the main flow rate. The whole cell system is immersed horizontally in a large vessel filled with an electrolyte solution. When direct current $i$ flows in the $y$-direction and a uniform external magnetic field $B$ is applied in the $z$ direction, a flow of the electrolyte solution arises along the channel (in the $x$-direction) due to the electromagnetic force.

Owing to the viscosity of the solution, boundary layers form on the channel walls. The thickness of the boundary layer is zero at the inlet but becomes larger as it goes toward the outlet. If the channel is short enough as in 


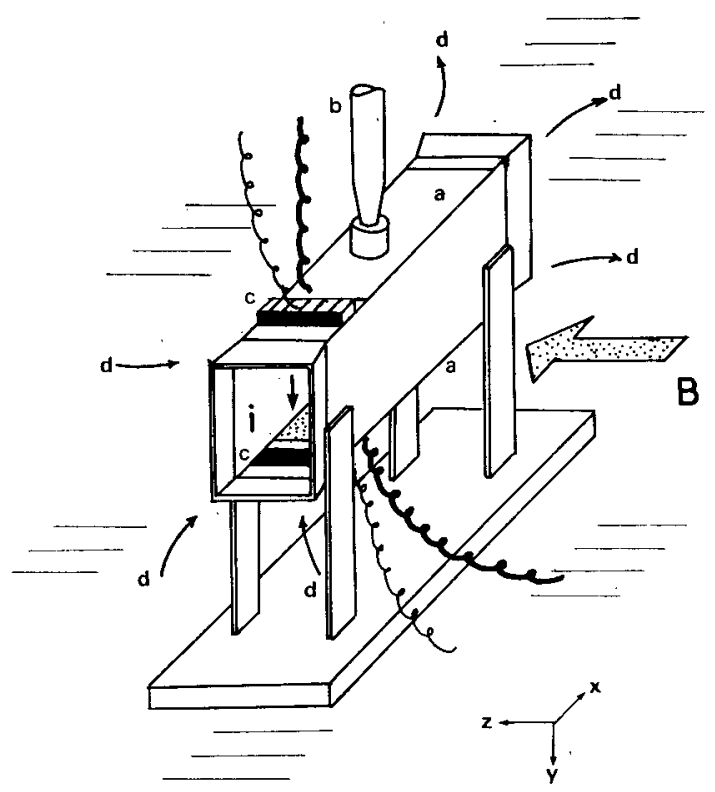

Fig. 1 Cell configuration

$\mathrm{a}:$ Working and counter electrodes, $\mathrm{b}:$ Luggin capillary, c : Electrodes for measuring main

flow rate, $d$ : Streamline

this case, and/or if the Reynolds number referring to the channel height is fairly large, the boundary layer region is trivial compared with the main flow region. Therefore, the hydrodynamic treatment can be made on two parts, boundary layer and main inviscid flow, separate$1 y$, as commonly used in hydrodynamics ${ }^{5}$. An incompressible flow induced by the electromagnetic force is expressed by the following Navier-Stokes and continuity equations,

$$
\begin{aligned}
& \frac{\partial \vec{v}}{\partial t}+(\vec{v} \cdot \nabla) \vec{v}=\nu \nabla^{2} \vec{v} \\
& \quad-\frac{1}{\rho} \nabla p+\frac{1}{\rho} \vec{i} \times \vec{B}, \\
& \nabla \cdot \vec{v}=0,
\end{aligned}
$$

where $\vec{v}$ : velocity, $t$ : time, $\nu$ : kinematic viscosity, $\rho:$ density, $p$ : pressure, $\vec{i}$ : current density, $\vec{B}$ : magnetic flux density. The electromagnetic force per unit volume is represented by the third term on the right side of Eq. (1) as the vector product of the externally applied current density, $\vec{i}$, and the external magnetic flux density, $\vec{B}$. In a strict sense, both the current and magnetic flux densities should be the real effective ones containing all terms caused by the induced phenomena, such as the voltage that arises from conductors moving in a magnetic field. But the approximations may be justified considering the insignificant secondary perturbations on the electric and magnetic fields due to the motion of the solution. This is because of the small ionic carrier mobilities in contrast to the large electron mobility which makes this secondary perturbation non-negligible in case of liquid metal systems. Figure 2 shows the streamlines of the solution in the vessel. As illustrated in the figure, the streamlines are approximately parallel to the wall surfaces inside the channel because of the extreamly thin boundary layers, while the streamlines gradually converge when the flow goes into the channel and they diverge when the flow goes out of the channel.

Equation (2), the continuity equation, results in the boundary conditions for the main flow as follows ;

$$
\begin{aligned}
\text { i) } \vec{v}=0 \text { as } x \rightarrow \pm \infty . \\
\text { ii) } v_{x}=U \text { (const.), } \\
v_{y}=v_{z}=0 \text { for } 0 \leqslant x \leqslant l .
\end{aligned}
$$

where $l$ is the channel length.

The electric current flows approximately in the electrode region $x_{1} \leqslant x \leqslant x_{2}$. Since the main flow is non-viscous, the Navier-Stokes equation

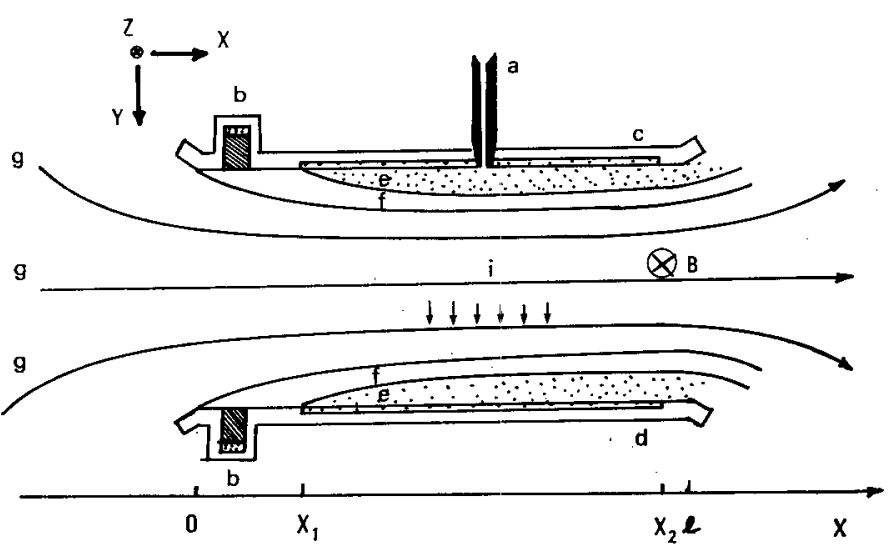

Fig. 2 Schematic profile of the cell and the solution flow a : Luggin capillary, $\mathrm{b}$ : Electrodes to measure the main flow rate, $c$ : Working electrode, $d:$ Counter electrode, e : Diffusion layer, $f:$ Hydrodynamic boundary layer, $\mathrm{g}:$ Streamline 
of steady state is

$$
(\vec{v} \cdot \nabla) \vec{v}=-\frac{1}{\rho} \nabla p+\frac{1}{\rho} \vec{i} \times \vec{B} .
$$

Integration of Eq. (5) from $x=-\infty$ to $x=l$ results in

$$
\frac{1}{2} U^{2}=-\frac{1}{\rho} \Delta p_{0}+\frac{1}{\rho} B \int_{x_{1}}^{x_{2}} i \mathrm{~d} x,
$$

where $U$ is the rate of main flow in the channel, and $\Delta p_{0}$ is the pressure difference between the outlet of the channel and $x=-\infty$. Furthermore, the mean current density $\bar{i}$ can be expressed in terms of the total current $I$, the electrode length $x_{2}-x_{1}$ and the electrode width $w$, as $\bar{i} \equiv I /\left(x_{2}-x_{1}\right) w$. On the other hand, $I$ is given by

$$
I=w \int_{x_{1}}^{x_{2}} i \mathrm{~d} x,
$$

thus $\mathrm{Eq} .(6)$ is rewritten in the following form;

$$
\begin{aligned}
\frac{1}{2} U^{2} & =-\frac{1}{\rho} \Delta p_{0}+\frac{1}{\rho} \frac{B I}{w} \\
& =-\frac{1}{\rho} \Delta p_{0}+\frac{1}{\rho} B \bar{i}\left(x_{2}-x_{1}\right) .
\end{aligned}
$$

The above relationship will be examined experimentally.

\section{Experimental and Results}

\subsection{Calibration of mhd flow meter}

In prior to perform the measurement of flow rate, the applicability of an $m h d$ flow meter to the present system was examined experimentally. When a uniform magnetic field is applied in the $z$-direction, and the electrolyte solution flows at a rate $v_{x}$ in the $x$-direction, emf, $V$,

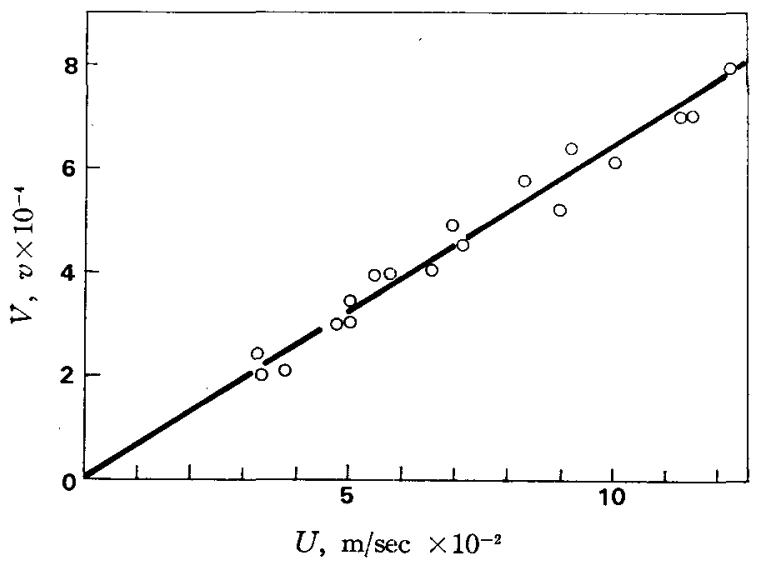

Fig. 3 Calibration curve of the induced emf against the flow rate represented by the following expression is generated in the $y$-direction.

$$
V=\int_{0}^{h} v_{x} B \mathrm{dy}=\bar{v} h B,
$$

where $\bar{v}$ is the mean flow rate in the $x$-direction, and $h$ is the height of channel. A rectangular channel, $1 \times 1 \mathrm{~cm}^{2}$ in cross-sectional area, was used for the $e m f$ measurement. A pair of copper electrodes, $0.5 \times 1 \mathrm{~cm}^{2}$ each, was $\mathrm{e}$ quipped to the channel and covered with agar-sol solutions of the same electrolyte composition as in the bulk in order to prevent the fluctuation of emf, $V$. A reservoir containing an aqueous solution of $0.2 . M \mathrm{CuSO}_{4}-1 M \mathrm{H}_{2} \mathrm{SO}_{4}$ was placed at a higher level than the channel in order to force the solution to flow, and connected with the channel through a plastic tube. The flow rate was determined by the volume of the liquid which passed the channel in a given time. $E m f$ was measured by means of a high input impedance recorder, Yokogawa 3048. The imposed magnetic flux density was $0.60 \mathrm{~Wb} / \mathrm{m}^{2}$, and the gap and radius of the cores of the electromagnet were $4.0 \mathrm{~cm}$ and $12 \mathrm{~cm}$, respectively.

Circles in Fig. 3 give the observed $U-V$ relation and the solid line shows the values calculated from Eq. (10). Agreements between the observed and calculated values were good. This result indicates that the main flow rate can be determined by the measurement of the

\begin{tabular}{|c|c|c|c|c|c|c|c|c|}
\hline $\begin{array}{c}\text { Cell } \\
\text { type }\end{array}$ & $\mathrm{h}$ & $x_{2}-x_{1}$ & a & $b$ & c & $\mathrm{d}$ & $\mathrm{w}$ & $r$ \\
\hline$\# 1$ & 5 & 5 & 1 & 0.5 & 3 & 0.5 & 1 & 0.118 \\
\hline \multirow{2}{*}{$\# 2$} & 2.5 & 5 & 0.2 & 0.5 & & 20.28 & 1 & \multirow{2}{*}{0.421} \\
\hline & \multicolumn{7}{|c|}{ unit, $\times 10^{-2} \mathrm{~m}$} & \\
\hline
\end{tabular}
induced $e m f$.

3.2 Electromagnetic force and main flow As shown in Figs. 1 and 2, another pair of

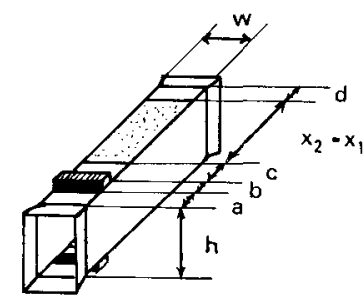

Table 1 Cell dimensions 
electrodes were attached as the flow detection electrodes, not on the down-stream side but on the upper-stream side of the main electrodes to avoid the interruption of the concentration overvoltage of the reaction. The structure of these detection electrodes was the same as used in the calibration experiments. The dimensions of the cell are given in Table 1.

The whole cell system was horizontally immersed in a large volume of a $0.5 \mathrm{MCuSO}_{4}-0.5$

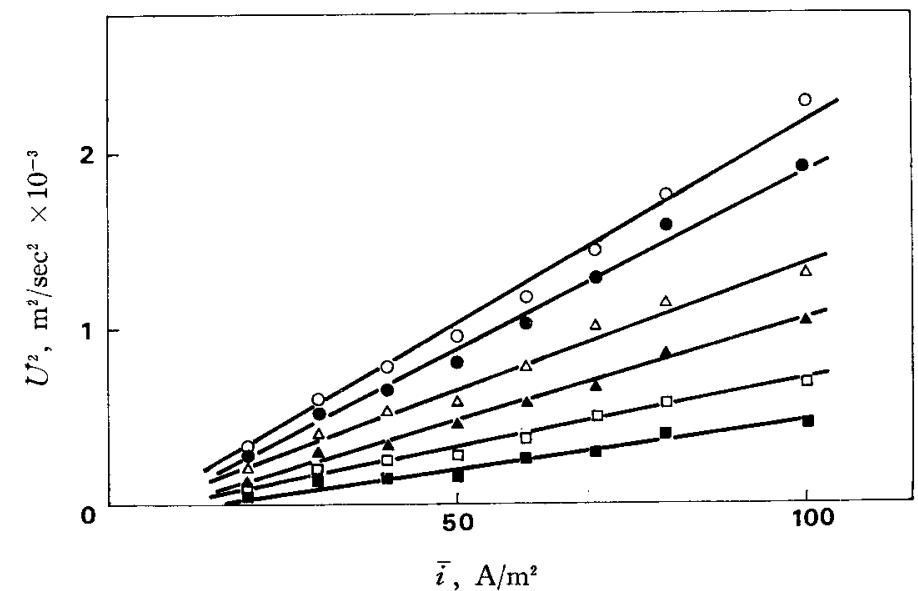

Fig. 4 Relation between the mean current density and the evaluated main flow rate at fixed magnetic flux density for the cell \#2

$O: B=0.6 \mathrm{~Wb} / \mathrm{m}^{2}$, $: B=0.5 \mathrm{~Wb} / \mathrm{m}^{2}, \quad \triangle: B=0.4$ $\mathrm{Wb} / \mathrm{m}^{2}, \quad \Delta: B=0.3 \mathrm{~Wb} / \mathrm{m}^{2}, \quad \square: B=0.2 \mathrm{~Wb} / \mathrm{m}^{2}$, 口: $B=0.1 \mathrm{~Wb} / \mathrm{m}^{2}$

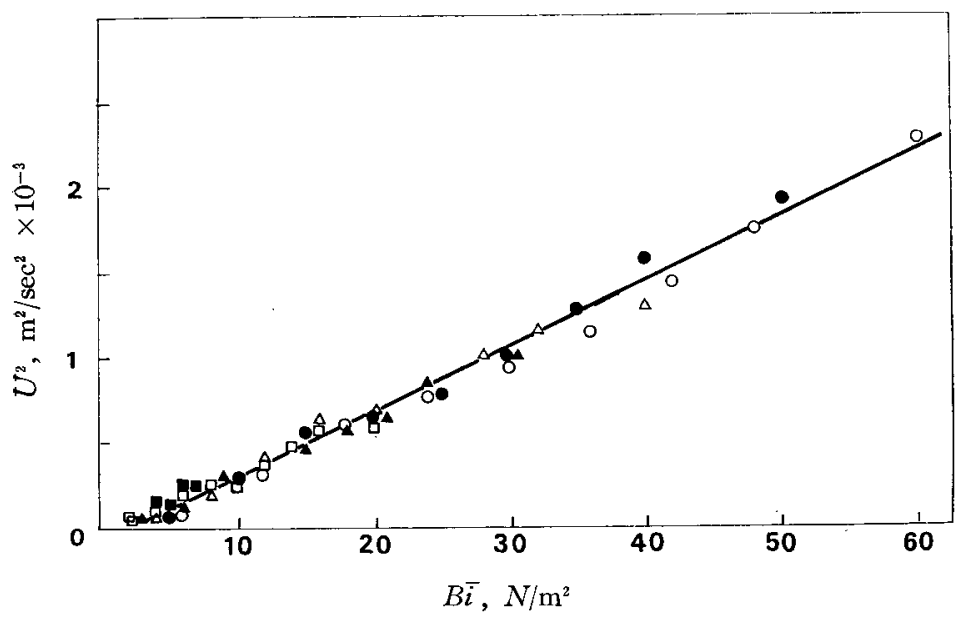

Fig. 5 Plot of the main flow rate vs. the electromagnetic force per unit volume for the cell $\neq 2$

$O: B=0.6 \mathrm{~Wb} / \mathrm{m}^{2}, \quad: B=0.5 \mathrm{~Wb} / \mathrm{m}^{2}, \triangle: B=0.4 \mathrm{~Wb} / \mathrm{m}^{2}$, $\Delta: B=0.3 \mathrm{~Wb} / \mathrm{m}^{2}, \square: B=0.2 \mathrm{~Wb} / \mathrm{m}^{2}, \boldsymbol{\square}: B=0.1 \mathrm{~Wb} / \mathrm{m}^{2}$
$M \mathrm{H}_{2} \mathrm{SO}_{4}$ solution, and a magnetic field was imposed in the $z$-direction. Then the solution was forced to flow magnetohydrodynamically by applying constant $d c$ current between the main pair of electrodes. The emf due to the fluid motion was obtained as the einf difference between two cases where the magnetic field was present and absent, and the main flow rate was determined using Eq. (10). The relationship between the square of velocity, $U^{2}$ and the mean current density, $\bar{i}$ at a fixed magnetic flux density, $B$ for the cell $\# 2$ shown in Table 1 is illustrated in Fig. 4.

A good linearity is observed between $U^{2}$ and $\bar{i}$. The plot of $U^{2}$ against the electromagnetic force per unit volume $B \vec{i}$ for the cell $\neq 2$ is shown in Fig. 5. Also a good linearity is seen.

\section{Discussion}

From the viewpoint of electrochemical analysis, the establishment of the main flow rate in the channel is equivalent to know, for instance, the rotation rate of the rotating disc electrode. This plays a quite important role in determination of the thickness of the boundary layer through which diffusional mass transport occurs. In consideration of the rate control method of the cell system, the relative advantages of this $m h d$ pump electrode system for electrochemical analysis may be in the following; (1) the reference electrode can be placed much closer to the working electrode because of the static nature of the electrodes, (2) the whole electrode system can readily be sealed under desired atmospheres, which is especially useful for non-aqueous electro- 
lyte solutions, and (3) it is the most convenient method to electrochemically analyse the change in the reactions under a magnetic field which is being paid the current attention ${ }^{6)-8)}$.

Although it has been shown that $U^{2}$ is in proportion to $B \bar{i}$ and the first term on the right side of Eq. (9) is negligible under proper conditions in the experiments, the absolute magnitude of $U^{2}$ was observed smaller than that predicted by Eq. (9). This is due to the several non-ideal factors associated with the real system. Some examples may be non-ideal streamlines broadening in the bulk owing to the finite volume of the vessel filled with the solution and non-ideal current distribution. For the practical purposes, therefore, Eq. (9) should be used in the form;

$$
-\frac{1}{2} U^{2}=\frac{\gamma}{\rho} B \bar{i}\left(x_{2}-x_{1}\right)
$$

where $r$ is a cell constant of the order of unity. Experimentally $r$ was the closer to unity as the ratio $h /\left(x_{2}-x_{1}\right)$ decreased and was constant for each cell.' Namely, it was 0.118 for the cell $\# 1$ of which the ratio $h /\left(x_{2}-x_{1}\right)=1$, and 0.421 for the cell $\$ 2$ with $h /\left(x_{2}-x_{1}\right)=0.5$. For the practical application of this mhd pump electrodes to electrochemical analysis, the linearity between $U^{2}$ and $\overline{B i}$ is of the essential significance to characterize the electrode process.

\section{Conclusions}

A quantitative magnetohydrodynamic treatment was made to predict the main potential flow rate of an electrolyte solution in a short channel between a pair of electrodes as a function of the current and magnetic field. The results obtained may be expressed as

$$
\frac{1}{2} U^{2}=-\frac{1}{\rho} \Delta p_{0}+\frac{1}{\rho} B \bar{i}\left(x_{2}-x_{1}\right) \text {. }
$$

Experimental observations indicated that the equation can be approximated for the practical purposes as

$$
\frac{1}{2} U^{2}=\frac{1}{\rho} \gamma \hat{B i}\left(x_{2}-x_{1}\right),
$$

with $\gamma$ being the cell constant. That is, the square of main flow rate is in proportion to magnetic flux density and mean electrolytic current density.

Acknowledgements :

The authors wish to express their hearty thanks to Dr. K. Kitazawa for his helpful discussion on the present work.

\section{Nomenclature :}

$\vec{v}:$ Velocity, $\mathrm{m} / \mathrm{s}$

$t:$ Time, $\mathrm{s}$

$\nu:$ Kinematic viscosity, $\mathrm{m}^{2} / \mathrm{s}$

$\rho:$ Density, $\mathrm{kg} / \mathrm{m}^{3}$

$p$ : Pressure, $N / \mathrm{m}^{2}$

$B$ : Magnetic flux density, $\mathrm{Wb} / \mathrm{m}^{2}$

$U$ : Main flow rate in the channel, $\mathrm{m} / \mathrm{s}$

$x_{1}, x_{2}: x$-coordinate of the edge of electrode, $\mathrm{m}$

$w:$ Electrode width, $\mathrm{m}$

$h:$ Distance between electrodes, $\mathrm{m}$

$l$ : Channel length, $\mathrm{m}$

$i$ : Electrolytic current density, $A / \mathrm{m}^{2}$

$\bar{i}$ : Mean current density; $A / \mathrm{m}^{2}$

$I$ : Total current, $A$

$\Delta p_{0}$ : Pressure difference between the outlet of the channel and $x=-\infty, N / \mathrm{m}^{2}$

$V:$ Induced $e m f, V$

$r:$ Cell constant

$<\mathrm{E} 415>$ (Received March 25, 1975)

\section{References:}

1) A.V. Leont'ev, A.G. Smirnov, Vopr. Magnith. Gidrodinam., Akad. Nauk Latv. SSr, Inst. Fiz. 3, 357 (1963).

2) N.I. Pekhteleva, A.G. Smirnov, ibid. 2, 89 (1965).

3) S. Mohanta, T.Z. Fahidy, Can. J. Chem. Engng. 50, 248 (1972).

4) T.Z. Fahidy, Electrochim. Acta 18, 607 (1973).

5) H. Schlichting, Boundary-Layer Theory, 6 th edn, Chap. VII, p. 117 (1968), McGRAW-HILL, New York.

6) L.R. Faulkner, A.J. Bard, J. Amer. Chem. Soc. 91, 209 (1969).

7) R.C. Johnson, R.E. Merrifield, Phys. Rev. B 1, 896 (1970).

8) K.S.V. Santhanam, Can. J. Chem. 49, 3577 (1971).

9) L.R. Faulkner, H. Tachikawa, A.J. Bard, J. Amer. Chem. Soc. 94, 691 (1972). 